\title{
Generalized synchronization in directionally coupled systems with identical individual dynamics
}

\author{
J. M. González-Miranda \\ Departamento de Fisica Fundamental, Universidad de Barcelona, Avenida Diagonal 647, 08028 Barcelona, Spain
}

(Received 13 November 2001; published 11 April 2002)

\begin{abstract}
A simple chaotic flow is presented, which when driven by an identical copy of itself, for certain initial conditions, is able to display generalized synchronization instead of identical synchronization. Being that the drive and the response are observed in exactly the same coordinate system, generalized synchronization is demonstrated by means of the auxiliary system approach and by the conditional Lyapunov spectrum. This is interpreted in terms of changes in the structure of the system stationary points, caused by the coupling, which modify its global behavior.
\end{abstract}

DOI: 10.1103/PhysRevE.65.047202

PACS number(s): 05.45.Xt, 05.45.Pq, 05.45.Ac

Synchronization of chaos is an issue of major interest in nonlinear dynamics. A significant result is the discovery of a variety of different synchronization phenomena that include identical synchronization (IS), and generalized synchronization (GS) among others [1]. Such variety of chaos synchronization scenarios raises questions regarding fundamental issues such as the conditions for the occurrence of the different phenomena. The present paper addresses GS, which is a phenomenon defined in the frame of directionally coupled chaotic systems, and refers to the situation when there is a continuous mapping that allows to define the trajectory of the response from the trajectory of the drive [2]. This form of synchronization, which includes identical synchronization [3] as a particular case, was introduced and has been studied $[1,2,4,5]$ as a form of synchronization proper of chaotic systems with nonidentical individual dynamics. IS is then seen as the form of synchronization proper of identical chaotic systems, while GS is understood as its generalization to deal with nonidentical systems. In the present paper, it will be shown that nonidentity of the systems is not a necessary condition for GS, because identical systems are also able to exhibit nontrivial forms of GS. This will be done by means of the example of two identical three-dimensional fluxes coupled directionally and monitored in exactly the same coordinate system; which, however, display GS combined with IS, depending on initial conditions. The origin of GS will be traced by a qualitative analysis of the global dynamics of the coupled six-dimensional system. Moreover, because of the existence of different basins of attraction, these results will provide an example of a case in which the test for GS known as the auxiliary system approach may fail to detect GS as warned, although not demonstrated, in Ref. [4].

Synchronization under a drive-response scheme is studied here. The drive system is an autonomous three-dimensional flow, $\mathbf{x}=\left(x_{1}, x_{2}, x_{3}\right)$, whose dimensionless equations of motion are

$$
\begin{gathered}
\dot{x}_{1}=x_{2}+3.2 \sin \left(1.4 x_{2}\right), \\
\dot{x}_{2}=-x_{2}-\left(x_{3}-R\right) x_{1}, \\
\dot{x}_{3}=x_{1}^{2}-x_{3},
\end{gathered}
$$

with $R$ a parameter of the model. This system was introduced to study the stability of IS in spatially symmetric chaotic systems and its use in communications [6]. In what follows we will take $R=5.2$, which makes the system chaotic with a Lyapunov spectrum $(0.13,0,-2.13)$. A plot of the chaotic attractor, given in Fig. 1, shows that the system dynamic behavior can be described as a coherent rotation in a simple chaotic band attractor. Local stability analysis shows that this system has three fixed points. One, at $\mathbf{X}^{(0)}=(0,0,0)$, is a saddle node, with eigenvalues $\lambda_{1}^{(0)}=-1, \lambda_{2}^{(0)} \approx-5.86$, and $\lambda_{3}^{(0)} \approx 4.86$. The respective eigenvectors are $\mathbf{e}_{1}^{(0)}=(0,0,1)$, $\mathbf{e}_{2}^{(0)} \approx(1,-1.07,0)$, and $\mathbf{e}_{3}^{(0)} \approx(1,0.89,0)$. The other fixed points are located at $\mathbf{X}^{(1,2)}=( \pm \sqrt{R}, 0, R)$, and are spiral-out saddles, with eigenvalues $\lambda_{1}^{(1,2)} \approx-4.54$ and $\lambda_{2,3}^{(1,2)} \approx 1.27$ $\pm i 3.31$, and eigenvectors $\mathbf{e}_{1}^{(1,2)}=(-0.59,0.49,-0.76)$, and $\mathbf{e}_{2,3}^{(1,2)} \approx(0.37 \pm i 0.92,-0.47 \pm i 0.44,-1.10 \mp i 0.25)$. These fixed points are displayed in Fig. 1. There, it is seen how the motion in the attractor results form the interplay between the spiral dynamics around the points $\mathbf{X}^{(1,2)}$ : a departing phase space point that rotates around $\mathbf{X}^{(2)}$, after having completed a bit more than a half rotation falls down onto the neighborhood of $\mathbf{X}^{(1)}$, this makes it to swirl around this point going

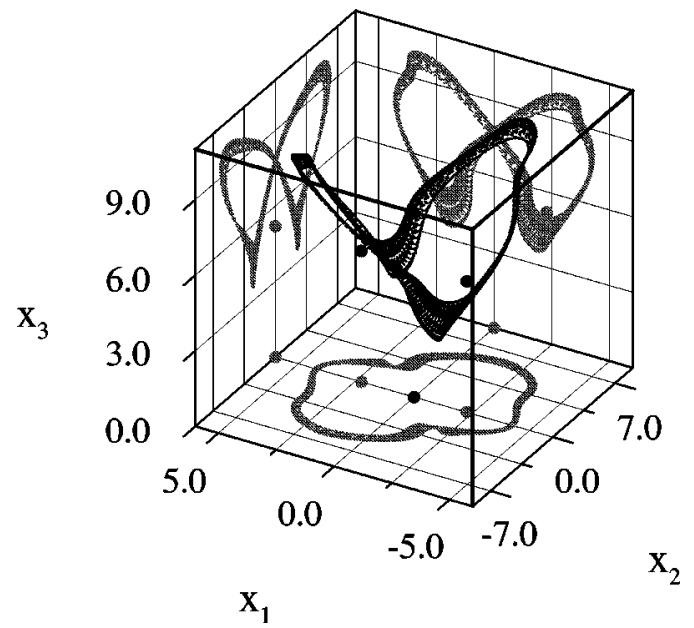

FIG. 1. Dynamic behavior of the flow given by Eqs. (1)-(3) for $R=5.2$. The stationary points appear as filled circles. Trajectories and points appear in black, and their projections onto the coordinate planes are plotted in gray. All quantities plotted are dimensionless. 

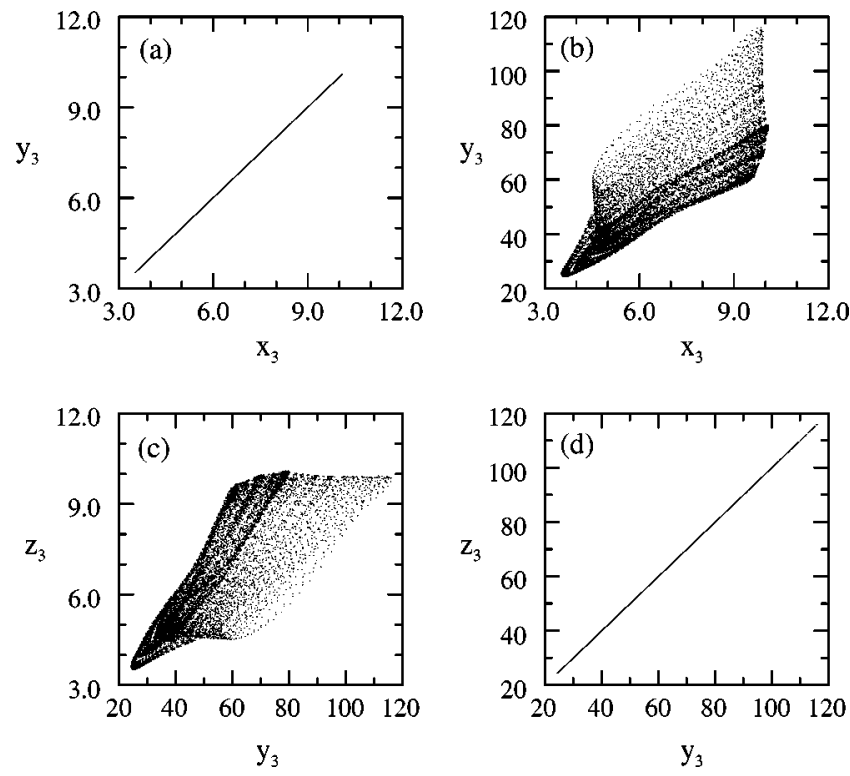

FIG. 2. Test for IS using initial conditions $\mathbf{x}_{D}^{(0)}$ for the drive, and (a) $\mathbf{y}_{a}^{(0)}$, or (b) $\mathbf{y}_{b}^{(0)}$, for the response. Auxiliary system approach test for GS when $\mathbf{y}_{b}^{(0)}$ is used for the initial conditions of the response, and (c) $\mathbf{z}_{a}^{(0+)}$, or (d) $\mathbf{z}_{b}^{(0)}$ for the auxiliary system. All quantities plotted are dimensionless.

over a new arc back to the influence of $\mathbf{X}^{(2)}$. The whole dynamics results from a back and forth motion between these two repellors. The fixed point at $\mathbf{X}^{(0)}$ acts as a repellor when the phase space trajectories approach their lower $x_{3}$ values.

In Ref. [6] the focus was on IS and symmetry. However, there are other dynamic behaviors available that will be show here to be a case of GS in directionally coupled systems with identical individual dynamics. For this aim, we consider the response system, $\mathbf{y}=\left(y_{1}, y_{2}, y_{3}\right)$, with

$$
\begin{gathered}
\dot{y}_{1}=y_{2}+3.2 \sin \left(\begin{array}{ll}
1.4 & y_{2}
\end{array}\right), \\
\dot{y}_{2}=-y_{2}-\left[x_{3}-R\right] y_{1}, \\
\dot{y}_{3}=y_{1}^{2}-y_{3},
\end{gathered}
$$

which generalizes the driving scheme studied in Ref. [6] because here a full copy of the drive is used instead of a part of it [3]. According to the literature, $[3,7]$, this sort of coupling, being the systems identical, may produce IS. If this were the case, the full dynamics occurring in the six-dimensional space defined by the combined set of Eqs. (1)-(6) will collapse onto a manifold such that $x_{i}(t)=y_{i}(t)$, for $i=1,2,3$. Results of a study of that possibility are given in Figs. 2(a) and 2(b) for two realizations that differ on the set of initial conditions used: $\mathbf{x}_{D}^{(0)}=(-2.5,-2.6,4.2)$ for the drive, and $\mathbf{y}_{a}^{(0)}=(3,0,0)$ or $\mathbf{y}_{b}^{(0)}=(10,0,0)$, respectively, for the response. In these plots and other similar that follow, only the third component of each subsystem is displayed because the plots for the other components would provide no new information. Figure 2(a) displays a clean straight line with slope equal to one, after a short transient (not shown), while Fig. 2(b) looks cloudy despite $10^{4}$ cycles after $t=0$ have been neglected before starting to plot. So one obtains IS only for certain initial conditions but not for all.

To study the case when no IS was observed the auxiliary system approach (ASA), which is a test for GS introduced by Abarbanel, Rulkov, and Sushchik [4] was used. For that aim an auxiliary system, $\mathbf{z}=\left(z_{1}, z_{2}, z_{3}\right)$, was constructed, given by

$$
\begin{gathered}
\dot{z}_{1}=z_{2}+3.2 \sin \left(\begin{array}{ll}
1.4 & z_{2}
\end{array}\right), \\
\dot{z}_{2}=-z_{2}-\left[x_{3}-R\right] z_{1}, \\
\dot{z}_{3}=z_{1}^{2}-z_{3} .
\end{gathered}
$$

According to Ref. [4] if the dynamics of the ninedimensional system given by Eqs. (1)-(9) collapses to a manifold that verifies $y_{i}(t)=z_{i}(t), i=1,2,3$; then, there is GS of the drive to the response in the sense that there is a transformation, $\mathbf{y}(t)=\phi[\mathbf{x}(t)]$, that gives the dynamic of the response from the dynamics of the drive, with $\phi[\cdot]$ a locally continuous, point to point, non-time-dependent transformation. This test for GS, which is partly shown in Fig. 2, displays different outputs depending on the initial conditions used for the response $\left[\mathbf{y}_{a}^{(0)}\right.$ or $\left.\mathbf{y}_{b}^{(0)}\right]$ and for the auxiliary system $\left[\mathbf{z}_{a}^{(0)}=(0,8,0)\right.$ or $\left.\mathbf{z}_{b}^{(0)}=(6,8,0)\right]$. For $\mathbf{y}_{a}^{(0)}$, one obtains that for $\mathbf{z}_{a}^{(0)}$ there is IS, which is a trivial case of GS, while for $\mathbf{z}_{b}^{(0)}$ ASA fails to detect synchronization. The corresponding plots are identical to Figs. 2(a) and 2(b), and, therefore, not displayed. The test for $\mathbf{y}_{b}^{(0)}$, [Figs. 2(c) and 2(d)], provides an indication that is reversed respect to $\mathbf{y}_{a}^{(0)}$ : no synchronization is detected for $\mathbf{z}_{a}^{(0)}$, and a proper case of GS for $\mathbf{z}_{b}^{(0)}$. In this last case the ranges of variation of $y_{3}$ and $z_{3}$ are different and disjoint from those observed for IS. Moreover, Fig. 2 suggests that $\phi[\cdot]$ is a complicated function. Some insight on the nature of $\phi[\cdot]$ can be obtained from a look to the plot of the trajectory of the response given in Fig. 3(a) for $\mathbf{y}_{b}^{(0)}$. While a plot for $\mathbf{y}_{a}^{(0)}$ will be identical to that given in Fig. 1 for the drive, the plot for $\mathbf{y}_{b}^{(0)}$ in Fig. 3(a) shows a response trajectory that is roughly an amplified version of the drive with a broader band structure. These results suggest that, in the six-dimensional space of initial conditions for $\mathbf{x}$ and $\mathbf{y}$, there are two basins of attraction one leading to IS, and other to GS. When both the response and the auxiliary systems are started at the same basin of attraction, ASA detects synchronization, which may be IS or GS. When the initial conditions of these two systems are in different basins, the ASA test fails to detect the synchronized states [4].

Another test for GS, given by Kocarev and Parlitz [5], has been done computing the conditional Lyapunov exponents for the response. The average values of the conditional Lyapunov exponents have been obtained over trajectories initiated at, $\mathbf{x}_{D}^{(0)}$, for the drive, and $\mathbf{y}_{a}^{(0)}$ or $\mathbf{y}_{b}^{(0)}$ for the response. According to Ref. [5], GS occurs if and only if all the exponents are negative. The conditional Lyapunov spectra obtained are $(-0.42,-0.58,-1.00)$ for $\mathbf{y}_{a}^{(0)}$ and $(-0.25$, $-0.76,-1.00)$ for $\mathbf{y}_{b}^{(0)}$. Therefore, in the two cases we have 

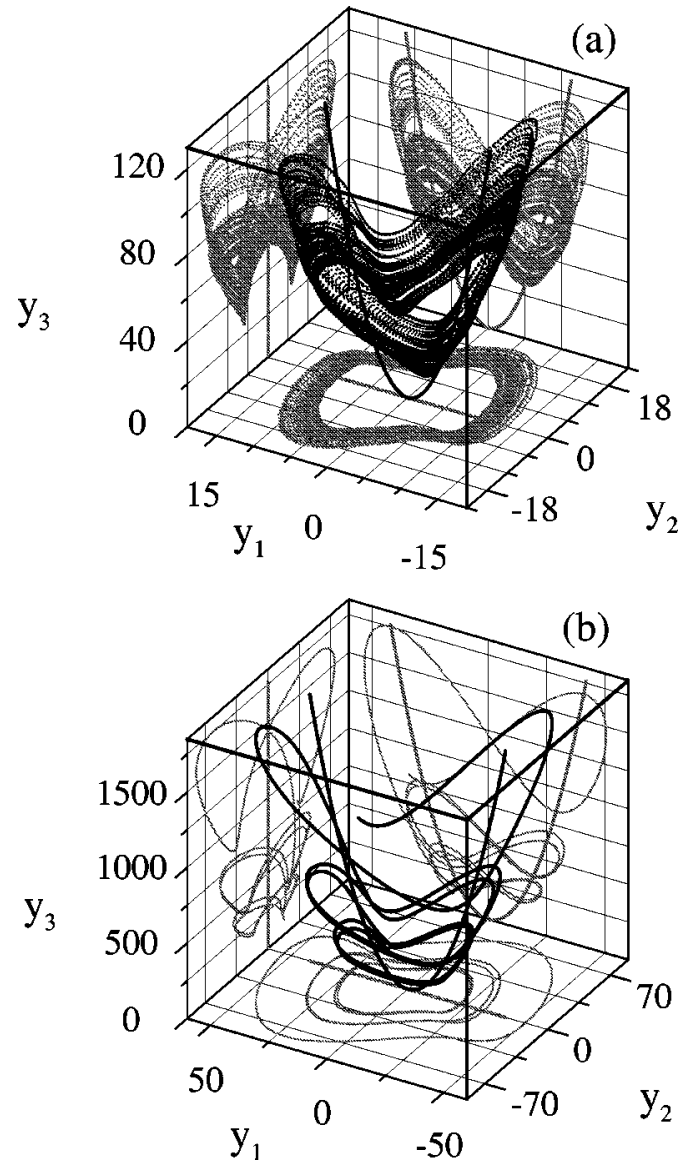

FIG. 3. Dynamic behavior of the response for initial conditions (a) $\mathbf{y}_{b}^{(0)}$, and (b) $\mathbf{y}_{c}^{(0)}=(15,8,100)$. The drive was started at $\mathbf{x}_{D}^{(0)}$. The set of points given by the parabola $y_{3}=y_{1}^{2}$ that is discussed in the text is also displayed. All quantities plotted are dimensionless.

asymptotically stable synchronized trajectories, being the absolute value of the largest conditional Lyapunov exponent for GS smaller than for IS.

The six-dimensional system given by Eqs. (1)-(6) has one fixed point at the origin, $\mathbf{X}^{(0)} \otimes \mathbf{Y}^{(0)}=(0,0,0) \otimes(0,0,0)$ and an infinite set of couples of fixed points given by $\mathbf{X}^{(1,2)}$ $\otimes \mathbf{Y}^{(1,2)}\left(y_{3}\right)=( \pm \sqrt{R}, 0, R) \otimes\left( \pm \sqrt{y_{3}}, 0, y_{3}\right)$. Because of the unidirectional nature of the coupling, the stability analyses of these points, can be factorized into two three-dimensional problems, one for the drive and another for the response. For the drive one obtains, as it must be, the same global behavior, within its own subspace, as for the uncoupled system. For the response, it results that $\mathbf{Y}^{(0)}=(0,0,0)$ has exactly the same eigenvalues and eigenvector as that of $\mathbf{X}^{(0)}$, and then has the same effect on the dynamics in the response subspace. The relevant points here are $\mathbf{Y}^{(1,2)}\left(y_{3}\right)$ that have eigenvalues -1 and 0 for any positive value of $y_{3}$, being the former eigenvalue degenerate. Therefore, $\mathbf{X}^{(1,2)} \otimes \mathbf{Y}^{(1,2)}\left(y_{3}\right)$ are still repellors in the full drive-response phase space, having a spiral-out saddle structure in the drive subspace, while in the response subspace there are two manifolds: one is stable and two dimensional, and the other is marginally stable, one dimensional, and tangent to the eigenvector $\left(1,0, \pm 2 \sqrt{y_{3}}\right)$ of $\lambda_{3}^{(1,2)}$ at each point $\left( \pm \sqrt{y_{3}}, 0, y_{3}\right)$. The tra- jectories in the response subspace tend to drop onto the points of the attracting curve of stationary points $y_{1}= \pm \sqrt{y_{3}}$, but the driving act prevents them to do so, and as a result they evolve around that curve. This is indeed what has been observed as mentioned previously, where we have obtained two separated attractors following this kind of motion (one for IS and other for GS), and response evolutions, as shown in Fig. 3(b), that spiral outwards following a curve around $\mathbf{X}^{(1,2)} \otimes \mathbf{Y}^{(1,2)}\left(y_{3}\right)$ that mimics the trajectory of the drive at ever increasing values of $y_{3}$.

The synchronization behavior cannot be inferred only from local stability arguments because a drive-response coupling does not warrants synchronization. According to the literature on synchronization of identical systems $[3,8]$ there is a basin of initial conditions leading to IS only when the largest conditional Lyapunov exponent is negative. When this happens, the response phase space points having coordinate values close to coordinate values of points of the drive attractor will follow trajectories that lead them to reproduce the dynamics of the drive. In other words, the drive trajectory is an attractor for the response. In the present case this exponent is $\Lambda_{1}=-0.42$; therefore, the response will evolve around its stationary points $\mathbf{Y}^{(1,2)}\left(y_{3}=R\right)$, i.e., the points $( \pm \sqrt{R}, 0, R)$ of the response subspace, in IS to the drive, for some initial conditions. However, for $y_{3}$ sufficiently greater than $R$ (let us say $y_{3}>y_{3}^{(m)}$ for certain $y_{3}^{(m)}>R$ ), there is a competition between two attractions: for one side there is a continuous segment of stationary points $\mathbf{Y}^{(1,2)}\left(y_{3}\right)$ with $y_{3}^{(m)}<y_{3}<y_{3}^{(M)}$ (being $y_{3}^{(M)}$ to be determined in the next paragraph), and for the other there is the drive trajectory. The balance these two attractions results in the GS attractor.

We have that for motions in the response subspace regions, the dynamics of the distance squared $s^{2}=y_{1}^{2}+y_{2}^{2}$ $+y_{3}^{2}$ will be given by

$$
d s^{2} / d t=y_{1}^{2} y_{3}+B\left(x_{3}\right) y_{1} y_{2}-\left(y_{2}^{2}+y_{3}^{2}\right)+y_{1} A \sin \left(\Omega y_{2}\right) .
$$

Despite this expression being quite complicated, if we restrict $x_{3} \sim R$ (motion of the drive in its stable attractor), which allows $\left|B\left(x_{3}\right)\right|=\left|1+R-x_{3}\right| \sim 1$, we obtain that, if $\left|y_{1}\right|,\left|y_{2}\right|$, and $y_{3}$ are all very large $\left(\left|y_{1}\right|,\left|y_{2}\right|, y_{3} \gg R, A\right)$, because of the cubic term, which is positive for all $y_{3}>0$, it is $d s^{2} / d t>0$ and the distance $s$ is an overall increasing function of time. In particular, for phase space trajectories of the kind depicted in Fig. 3(a) following the drive attractor around $\mathbf{X}^{(1,2)} \otimes \mathbf{Y}^{(1,2)}\left(y_{3}\right)$, but with $y_{3}$ very large one has $d s^{2} / d t>0$ in wide regions of phase space and no stable motion is expected. This has been observed in the numerical simulation [Fig. 3(b)]. The GS attractor then results from the wandering of the response phase space points, following the drive, around two segments of stationary points $\mathbf{X}^{(1,2)}$ $\otimes \mathbf{Y}^{(1,2)}\left(y_{3}\right)$, with $y_{3}^{(m)}<y_{3}<y_{3}^{(M)}$, being $y_{3}^{(m)}$ large enough as to prevent them from dropping onto the IS attractor, and $y_{3}^{(M)}$ small enough as to avoid motions in regions of phase space where the response is unstable.

The dynamic behaviors described above have been found to be reproducible using other values for the initial conditions and different system parameters. However, to appreci- 
ate the extent of the occurrence of GS, it is interesting to have a view of the basins of attraction to the two types of synchronization available. For this aim four of the six coordinates that define a complete set of initial conditions were fixed, and values for the other two were taken in a rectangular grid chosen in a region around the stable attractor. Then, Eqs. (1)-(6) were evolved for each initial condition in the grid, and the output reached was tested by means of two criteria: from the ASA test it follows that if $9<\max \left(y_{3}\right)$ $<11$ we have IS, and if $100<\max \left(y_{3}\right)<120$ we have GS; otherwise, from the Lyapunov spectrum test, if $-0.45<\Lambda_{1}$ $<-0.40$ we have IS, and if $-0.22<\Lambda_{1}<-0.27$ we have GS. The basins of attraction obtained are exactly the same no matter which of these two criteria is used. A thorough study showed that, for fixed $\mathbf{x}^{(0)}$, the relevant structures appear in the $y_{1}^{(0)}-y_{2}^{(0)}$ plane, the particular shapes of these basins change when $\mathbf{x}^{(0)}$ changes, displaying, however, the same overall behavior: regions leading to IS, close to the origin, and regions leading to GS, around them. Moreover, there are regions of unstable motion far from the origin, where the response trajectories do spiral outwards to infinity. Example images of such basins are given in Fig. 4 for grids of 99 $\times 99$ points. All this is in accordance with the stability arguments given above.

Finally, I would like to mention that Maistrenko et al. [9] have observed that mutually coupled identical logistic maps have attractors, which are curves, different from the diagonal, $\mathbf{x}=\mathbf{y}$. Although they did not discuss their results from the point of view of synchronization, this suggest GS in identical systems in a case very different from the one studied here.

In conclusion, an example of a chaotic flow has been (a)
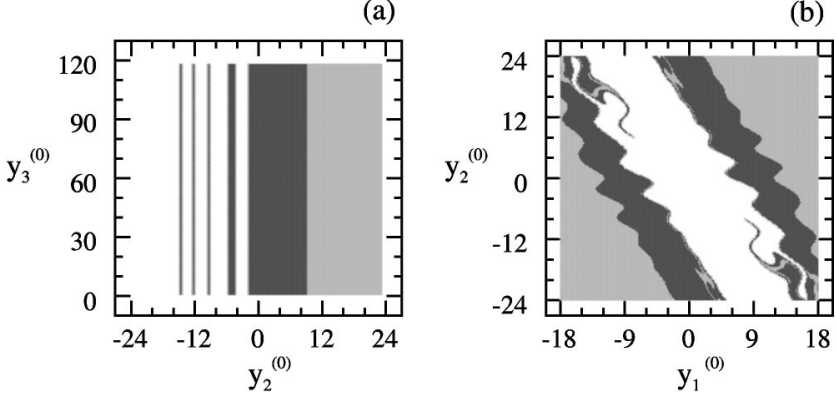

FIG. 4. Two-dimensional sections of the basins of attraction for $\mathbf{x}_{D}^{(0)}$, and the initial conditions of the response in the planes: (a) $y_{1}^{(0)}=9$, and (b) $y_{3}^{(0)}=0$. The color code is: white for IS, black for GS, and gray for unstable motions of the response. All quantities plotted are dimensionless.

given that displays a nontrivial form of GS in directionally coupled chaotic systems with identical individual dynamics that allow IS. This GS occurs for initial conditions in wide regions of the initial conditions six-dimensional space. Its origin has been associated with the appearance of new sets of stationary points, caused by the coupling, which happen to be attractive in the response subspace and compete with the attraction to the drive trajectory. This supports the assertion that nonidentity between drive and response is not a necessary condition for GS. Moreover, this system provides an illustration of how the auxiliary system approach has to be used with care to detect GS when there are two or more basins of attraction.

This research has been supported by DGI through Project No. BFM2000-0606.
[1] R. Femat and G. Solís-Perales, Phys. Lett. A 262, 50 (1999); R. Brown and L. Kocarev, Chaos 10, 344 (2000); S. Boccaletti, L.M. Pecora, and A. Peláez, Phys. Rev. E 63, 066219 (2001).

[2] N.F. Rulkov, M.M. Sushchik, L.S. Tsimring, and H.D.I. Abarbanel, Phys. Rev. E 51, 980 (1995).

[3] L.M. Pecora and T.L. Carroll, Phys. Rev. Lett. 64, 821 (1990).

[4] H.D.I. Abarbanel, N.F. Rulkov, and M.M. Sushchik, Phys. Rev. E 53, 4528 (1996).

[5] L. Kocarev and U. Parlitz, Phys. Rev. Lett. 76, 1816 (1996).
[6] J.M. González-Miranda, Phys. Rev. E 53, 5656 (1996); Phys. Lett. A 251, 115 (1999).

[7] J. Güémez and M.A. Matías, Phys. Rev. E 52, R2145 (1995).

[8] P. Badola, S.S. Tambe, and B.D. Kulkarni, Phys. Rev. A 46, 6735 (1992); M. de Sousa Vieira, A.J. Lichtenberg, and M.A. Lieberman, ibid. 46, R7359 (1992).

[9] Yu.L. Maistrenko, V.L. Maistrenko, A. Popovich, and E. Mosekilde, Phys. Rev. E 57, 2713 (1998). 